\title{
Vehicle Position Updating Strategy Based on Kalman Filter Prediction in VANET Environment
}

\author{
Yuanfu Mo, ${ }^{1}$ Dexin Yu, ${ }^{1}$ Jun Song, ${ }^{2}$ Kun Zheng, ${ }^{1}$ and Yajuan Guo ${ }^{1}$ \\ ${ }^{1}$ College of Transportation, Jilin University, Changchun 130022, China \\ ${ }^{2}$ Dalian International Airport, Dalian 116022, China
}

Correspondence should be addressed to Yuanfu Mo; 496629815@qq.com

Received 4 November 2015; Revised 10 December 2015; Accepted 13 December 2015

Academic Editor: Elmetwally Elabbasy

Copyright (C) 2016 Yuanfu Mo et al. This is an open access article distributed under the Creative Commons Attribution License, which permits unrestricted use, distribution, and reproduction in any medium, provided the original work is properly cited.

In VANET (vehicular ad hoc network) environment, the successive vehicle position data actually are discrete, so the key to the moving vehicle modeling is to effectively reduce the updating frequency of the position data so as to alleviate the communication and database management load. This paper proposes vehicle position data updating strategy with packet repetition based on Kalman filter predicting. Firstly, we design a position data updating model based on Kalman filter difference predicting equations. Then, we design a packet repetition mode decision algorithm, which is applied to deliver vehicle position updating data. The model with packet repetition can not only generate position updating data according to preset threshold, but also decide packet repetition mode related to the distance of two adjacent vehicles in order to reduce data loss. Both simulated highway and realistic urban road experimental results show that vehicle position data updating frequency could be obviously reduced and the reliability of the communication is greatly improved through packet repetition mechanism by using this position updating strategy.

\section{Introduction}

In VANET environment, the accurate position information of the moving vehicles in the road network could be obtained through the in-car mobile locating device (such as GPS), inertial measurement unit, and other in-car position sensors. Wireless communication facilities (On Board Unit, OBU, and Road Side Unit, RSU) can deliver real-time or near real-time position information to data center for more complex applications, such as real-time traffic navigation, traffic behavior studies, and location-related applications such as vehicle forward collision warning application based on central server [1].

Before implementing these applications, the first problem to solve is central management of the position data. Moving object database (MOD) is a branch of the database research field developed in recent years. It records the position of the different moving objects at different times [2]. The users can query the status of the moving objects from the database at a certain time [3]. In VANETs (vehicular ad hoc networks), the positions of the mobile vehicles changed frequently in a very short cycle time, such as the vehicles installed GPS receiver update positions every 10 seconds. Thus if the database is realtime refreshed, the bandwidth of communications and the database I/0 load will face enormous pressure [4]. Therefore, the key to mobile vehicle modeling is to reduce the updating frequency and make the continuous change become the discrete change [5]. Wolfson Research Group of American Illinois University in Chicago conducts the first study about the mobile target item and proposed the corresponding moving model MOST (moving objects spatial-temporal) to achieve position updating by storing the movement patterns of mobile objects (such as speed and direction) as dynamic properties [6]. Specifically, the position of the mobile object is abstracted as a function of time, and the calculated (predicted) value derived from the function is compared with the actual position. When the deviation of the both reaches a certain threshold, it is necessary to issue position updating request to update the database. This "prediction and comparison" method greatly reduces the load of communication and the pressure of data management, which is a fundamental principle of MOD position updating strategy. However, this model cannot achieve the server-based applications, such as the sky-server-based collision avoidance application [7]. 
Wu et al. [8] established an object-oriented all-time-domain data model for moving objects. The model added dynamic attributes to object-oriented model, which supported alltime-domain data storage and query. The model location updating threshold was given dynamically in accordance with the velocity, accuracy, and azimuth positioning information from the GPS. The above could realize the trajectory-based applications, but the applications based on prediction are not supported.

To date, coordinated techniques and probing vehicle method, which were employed to acquire and exchange of position data based on VANETs, have been thoroughly studied in the past few years. Xu et al. [9] revealed some intrinsic relationships between coordinated vehicle and road communication system of platoon safety by comparing different information structures and contents. Sekine [10] presented possibility of realizing safe mobility by equipping vehicles with probes. Amoroso et al. [11] presented a distributed multihop broadcast algorithm for vehicular safety, which is capable of reaching all vehicles with minimum number of transmissions with a realistic setting. Marfia et al. [12] conduct field trials and tested an accident warning system based on the multihop broadcast algorithm that has been proven to be optimal in terms of bandwidth usage and covered distance on realistic road. The above VANET-related safety studies are all based on distributed routing algorithm and exchanging relevant information by multihop broadcasting.

With the rapid development and application of big data and 5G mobile communication technology, it is a promising solution to store mass information in central database and direct transmits safety related messages to the destination node in form of single-hop broadcasting routing decided by central-server in VANET accident warning system. There are two main technical challenges associated with these centralserver-based accident avoidance systems [13]. The first is the generation of key point with respect to the reliable estimation of a vehicle's position in terms of reducing position data updating. The second is the reliable transmission of these position data through the channel to their intended recipients with high probability. This paper focuses on these two challenges, which reside in reducing vehicle position data updating frequency and improving reliability of the intervehicle's single-hop broadcasting. To achieve these two goals, we should research the position updating algorithms and strategies based on prediction threshold on the basis of the accurate and real-time predictive results, which could also ensure the timeliness and reliability of message delivery. Thus the position data updating pressure in central server could be reduced, and the robustness of data transmission is increased.

At first, this paper presents the vehicle position description model and then gives the predictive differential equations of position. On the basis of this, the vehicle position data updating model is designed and the repetition mode decision algorithm based on neighborhood distance is put forward to optimize the transmission part of the model. So the vehicle position data updating strategy is formed. Finally, the strategy is verified on both simulated highway and actual urban road.

\section{The Descripting Model of Vehicle Position in VANETs}

The vehicles as nodes in VANETs have a high degree of mobility with frequent position changes, and the distribution of vehicles is complex. Under normal circumstances, the vehicle is seen as a three-dimensional particle in free space in the dynamic model of the vehicle position. The navigation receivers of GPS have the accuracy of only 10 to 15 meters. So it is essential to use differential GPS (DGPS), if the positioning accuracy is increased to centimeter level. In order to compensate for the disruption of GPS signal error, we should do data fusion of the GPS, in-vehicle inertial measurement unit, and other in-vehicle sensors [14-16] to reduce the position drift error.

The vehicle position in VANETs can be represented as a vector $\vec{X}=(X, Y, V, \phi)^{T}$, where $X$ represents longitude, $Y$ represents latitude, $V$ represents velocity, and $\phi$ represents direction.

\section{The Vehicle Position Prediction Algorithm Based on Kalman Filter}

Kalman filter is a widely used prediction algorithm [17-19]. The basic idea can be stated as follows. The best estimate guideline is regarded as the minimum mean square error utilizing the state-space model of signal and noise. The estimated values of the previous and the observation values of the current are employed to update the estimation values of state variables. Thus an estimated value of the current time is obtained. According to the system and observation equations, the algorithm makes an estimate of the signal required to be processed meeting the minimum mean square error. Discrete Kalman filter equations include time updating equations (1) and state updating equations (2):

$$
\begin{aligned}
\widehat{X}_{k}^{-} & =A \bar{x}_{k-1}+B u_{k}, \\
P_{k}^{-} & =A P_{k-1} A^{T}+Q, \\
K_{k} & =P_{k}^{-} H^{T}\left(H P_{k}^{-} H^{T}+R\right)^{-1}, \\
\bar{x}_{k} & =\bar{x}_{k}^{-}+K_{k}\left(Z_{k}-H \bar{x}_{k}^{-}\right), \\
P_{k} & =\left(I-K_{k} H\right) P_{k}^{-} .
\end{aligned}
$$

In above equations, $A$ is the state transition matrix; $Q$ is the noise covariance matrix; $H$ is the observation matrix; and $R$ is the noise covariance matrix of the observation. Observation vector $Z_{k}$ is achieved from the current node, namely, the node's current position. $G_{k}$ is the stable gain matrix.

In this paper, the vehicle position vector is considered as the status vector of Kalman filter and the cycle is 10 seconds. Then the vehicle position is predicted after 10 seconds depending on the position vector and the estimation vector at current moment. 


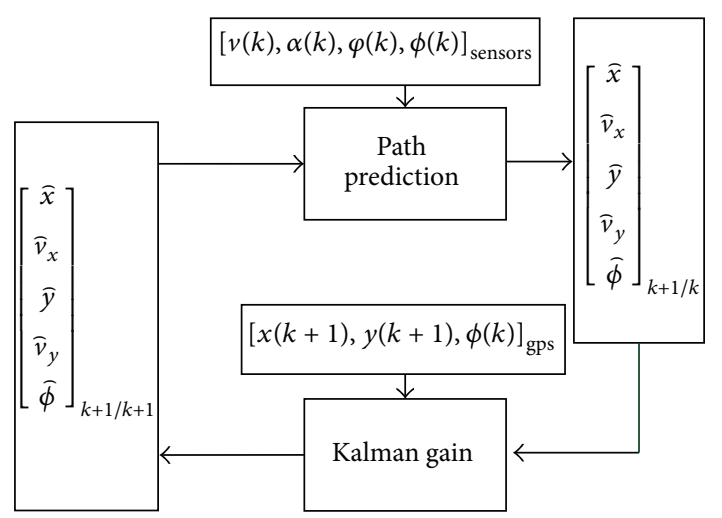

FIgURE 1: The vehicle position Kalman filter prediction model.

\section{The Differential Prediction Equations Based on Kalman Filter}

\subsection{Hypothesis}

(1) Each vehicle runs a Kalman filter predictor, which is employed to predict the vehicle's own position, velocity, and direction. Its position vector at moment $k$ is $\vec{x}(k)=[x(k), y(k), v(k), \phi(k)]^{T}$. Its predicted position vector at moment $k$ is $\overline{\vec{x}}(k)=$ $[\widehat{x}(k), \widehat{y}(k), \widehat{v}(k), \bar{\phi}(k)]^{T}$.

(2) The time difference of the moment $k$ and the moment $k+1$ is $\Delta T=T_{k+1}-T_{k}$.

The dynamics of a moving vehicle are sufficiently modeled by a few continuous-time, time-invariant, nonlinear equations. VANET-based applications consider wheel speeds, wheel angles, and vehicle positions as state variables. The measurements necessary for vehicle path prediction are available from sensors already available on many vehicles for vehicle status control purposes. Figure 1 shows the vehicle position prediction process. The measured value at moment $k$ from sensors is put into the path prediction block. The path prediction block uses the classic bicycle model to predict vehicle position at moment $k+1$. Variable $\alpha(k)$ represents steering angle, and variable $\varphi(k)$ represents heading of the vehicle at moment $k$. Equations (3) and (4) are path prediction equations. The predicted value from path prediction block is put into Kalman gain block, which combines path predicted value with GPS measured value at moment $k+1$. Equation (5) is the Kalman gain block. The path prediction equations are

$$
\begin{aligned}
& \widehat{x}(k+1)=\widehat{v}_{x}(k) \cos (\varphi(k))-\widehat{v}_{y}(k) \sin (\varphi(k)), \\
& \widehat{y}(k+1)=\widehat{v}_{x}(k) \sin (\varphi(k))+\widehat{v}_{y}(k) \cos (\varphi(k)) ; \\
& \widehat{v}_{x}(k+1)=f_{1}(v(k), \alpha(k), \Delta T, \widehat{\phi}(k), \dot{\phi}(k)) ; \\
& \widehat{v}_{y}(k+1)=f_{2}(v(k), \alpha(k), \Delta T, \widehat{\phi}(k), \dot{\phi}(k)) ; \\
& \widehat{\phi}(k+1)=\widehat{\phi}(k)+\dot{\phi}(k) \Delta T,
\end{aligned}
$$

where

$$
\begin{aligned}
& f_{1}(v(k), \alpha(k), \Delta T, \widehat{\phi}(k), \dot{\phi}(k)) \\
& =\frac{-2\left(C_{\alpha f}+C_{\alpha r} \alpha(k)\right) v_{y}(k)}{M v_{x}(k) \Delta T} \sin (\bar{\phi}(k)) \\
& +\left(\frac{-2\left(C_{\alpha f}-C_{\alpha r} l_{f}\right)}{M v_{x}(k)}-v_{x}(k) \Delta T\right) \cos (\widehat{\phi}(k)) \\
& -\left(\frac{2 C_{\alpha f} \alpha(k)}{M}\right) \dot{\phi}(k) \cos (\bar{\phi}(k)) \Delta T, \\
& f_{2}(v(k), \alpha(k), \Delta T, \widehat{\phi}(k), \dot{\phi}(k)) \\
& =\frac{-2\left(C_{\alpha f}+C_{\alpha r} \alpha(k)\right) v_{y}(k)}{M v_{x}(k) \Delta T} \cos (\bar{\phi}(k)) \\
& -\left(\frac{-2\left(C_{\alpha f}-C_{\alpha r} l_{f}\right)}{M v_{x}(k)}-v_{x}(k) \Delta T\right) \sin (\widehat{\phi}(k)) \\
& +\left(\frac{2 C_{\alpha f} \alpha(k)}{M}\right) \dot{\phi}(k) \sin (\bar{\phi}(k)) \Delta T,
\end{aligned}
$$

where $M$ represents vehicle mass, $\alpha$ represents steering angle, $\dot{\phi}$ represents heading rate, $\varphi$ represents heading of the vehicle, $l_{f}$ and $l_{r}$, respectively, represent the perpendicular distances from the front and rear axles to the vehicle's center of gravity, and $C_{\alpha f}$ and $C_{\alpha r}$, respectively, represent the linear front and rear tire cornering stiffness.

The Kalman gain equations are

$$
\widehat{X}_{k+1}=\widehat{X}_{k}+W_{k+1}\left(Z_{\text {gps }}(k+1)-\widehat{X}_{k}\right),
$$

where $Z_{\mathrm{gps}}=\left(x_{\mathrm{gps}}, y_{\mathrm{gps}}, \phi_{\mathrm{gps}}\right), W_{k+1}=P_{k} H_{k+1}^{T} S_{k+1}^{-1}, P_{k+1}=P_{k}-$ $W_{k+1} S_{k+1} W_{k+1}^{T}, H_{k+1}=\partial\left([\bar{x}, \widehat{y}, \widehat{\phi}]^{T}\right) /\left.\partial\left(\left[\bar{x}, \widehat{y}, \widehat{v}_{x}, \widehat{v}_{y}, \bar{\phi}\right]^{T}\right)\right|_{k+1}$, and $S_{k+1}=H_{k+1} P_{k} H_{k+1}^{T}+W_{k+1}^{T}$.

Then at moment $k+1$, the predicted values of the vehicle position vector $\vec{x}(k+1)=[x(k+1), y(k+1), v(k+1), \phi(k+1)]^{T}$ are as follows:

$$
\begin{aligned}
& \widehat{x}(k+1)=\widehat{x}(k)+\widehat{v}(k) \times \cos (\widehat{\phi}(k)) \times \Delta T ; \\
& \hat{y}(k+1)=\widehat{y}(k)+\widehat{v}(k) \times \sin (\widehat{\phi}(k)) \times \Delta T ; \\
& \widehat{v}(k+1)=\widehat{v}(k) ; \\
& \bar{\phi}(k+1)=\bar{\phi}(k)+\overline{\dot{\phi}}(k) \times \Delta T .
\end{aligned}
$$

In this paper we define (6) as Kalman filter difference prediction equations. The vehicle position information at moment $k+1$ could be predicted in accordance with the vehicle position at moment $k$ by (6). 


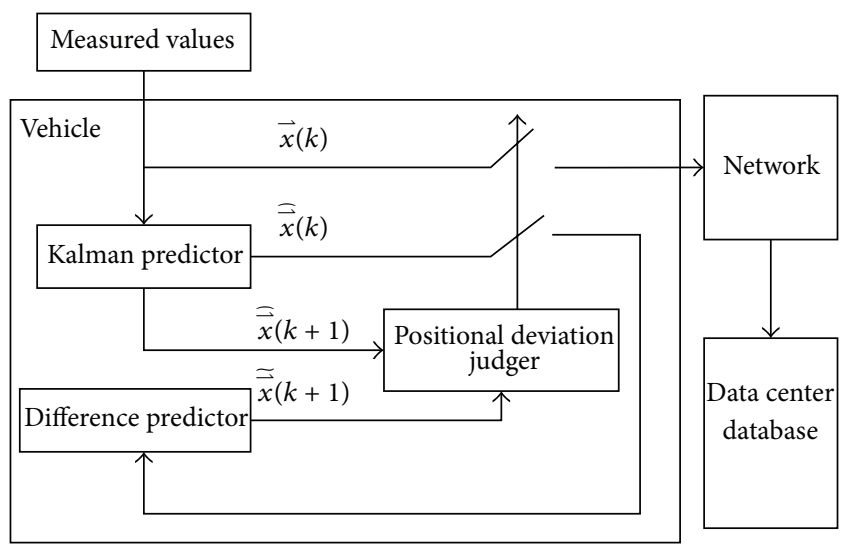

FIGURE 2: The vehicle position updating model.

\section{Vehicle Position Data Updating Strategy}

5.1. Vehicle Position Data Updating Model. As shown in Figure 2, the difference predictor is built on Kalman filter difference prediction equations mentioned above. According to the current vehicle measured value $\vec{x}(k)$ and predicted value $\overline{\bar{x}}(k)$, we get the Kalman predicted value $\overline{\bar{x}}(k+1)$ of next moment through Kalman filter predictor. The difference predicted value $\widetilde{\vec{x}}(k+1)$ of next moment can be gotten through current predicted value $\vec{x}(k)$ by difference predictor.

The values $\widehat{\vec{x}}(k+1)$ and $\widetilde{\vec{x}}(k+1)$ are put into the position deviation judgment; compared with the preset threshold, if they are more than threshold value, the vehicle position updating request is launched. Then vehicle current position $\vec{x}(k)$ is sent to the data center database server via VANET. At the same time the current moment position predicted value $\overline{\vec{x}}(k)$ is put into difference predictor for the next moment difference prediction.

Positional deviation judger is responsible for comparing the difference between $x$ and $y$ in vectors $\widehat{\bar{x}}(k+1)$ and $\widetilde{\vec{x}}(k+$ $1)$. The judgment equations are as follows:

$$
\begin{aligned}
\xi_{\text {long }} & =\mid(\widetilde{x}(k)-\bar{x}(k)) \times \cos (\widehat{\phi}(k))-(\tilde{y}(k)-\widehat{y}(k)) \\
\quad & \times \sin (\widehat{\phi}(k)) \mid ; \\
\xi_{\text {lat }} & =\mid(\widetilde{x}(k)-\bar{x}(k)) \times \sin (\widehat{\phi}(k))+(\tilde{y}(k)-\bar{y}(k)) \\
\quad & \times \cos (\widehat{\phi}(k)) \mid .
\end{aligned}
$$

In (7), $\xi_{\text {long }}$ is the vertical prediction deviation between the Kalman predictor and the difference predictor; $\xi_{\text {lat }}$ is the horizontal prediction deviation between the Kalman predictor and the difference predictor.

5.2. The Vehicle Position Data Updating Model with Packet Repetition. DSRC (Dedicated Short-Range Communication) is the basis of VANET, which is a kind of wireless communication system. DSRC links the vehicles and road organically through the two-way transmission of information. In VANET
DSRC wireless communication environment, packet loss is a factor that must be considered because it can bring vehicle position data error and delay in data center, thus affecting the central-server-based vehicle forward collision warning judgment in VANET active safety applications. PDR (Packet Delivery Ratio) refers to the total number of packets on the receiver than the packets from the sender of the ratio. PDR can not only reflect the maximum throughput which the wireless network can support, but also describe the integrity and correctness of the vehicle position data updating model to some extent [20]. Bai and Krishnan's [21] study shows that $P D R$ decreases with the increase of the communication distance between the vehicles. For example, in the highway environment, when the communication distance between two adjacent vehicles is 100 meters, the $P D R$ is $91 \%$. When the communication distance is 200 meters, the $P D R$ is $68 \%$. When the communication distance is 300 meters, the $P D R$ is $57 \%$. When the communication distance is up to 400 meters, the $P D R$ is $48 \%$.

In order to reduce packet loss that affects the vehicle position information data storage and updating in data center, this paper designs the vehicle position data updating model with packet repetition, which is shown in Figure 2.

In Figure 3, according to routing protocol determined by the central server, the vehicle obtains the desired communicating adjacent vehicle position data $\vec{y}(k)$ from the data center. According to the vehicle position $\vec{x}(k)$ and adjacent vehicle position $\vec{y}(k)$, Euclidean distance between two vehicles could be calculated. By the Euclidean distance of two vehicles, the repetition mode decider runs repetition algorithm to get the repetition times corresponding to position data $\vec{x}(k)$. Thus this position data will be delivered to the adjacent vehicle. The repetition mode decision algorithm is described in Algorithm 1.

In Algorithm 1, Eucli_Dist represents the Euclidean distance between two adjacent vehicles; Max Number_of_Repetitions represents the maximum number of repetitions; Max_Transmission_Dist represents the maximum allowable transmission distance; Adjust_PDR represents the expectation PDR; Min_Repetions_Dist represents the preset minimum transmission distance; Repetions_Interval represents the default repetition Interval; and Number_of_Repetitions represents the number of repetitions running this algorithm to get.

5.3. Vehicle Position Data Updating Strategy. At the moment $k$, if $\xi_{\text {long }}$ or $\xi_{\text {lat }}$ is more than the system preset threshold, the vehicle will initiate position updating request and the current observed value $\vec{x}(k)$ will be sent to the data center. The database stores new position information which is as a key point of vehicle position in MOD database. follows:

Judgment rules of position updating strategy are as

$$
\begin{aligned}
& \text { if }\left(\xi_{\text {long }}(k+1) \geq T r_{\text {.long. }} \vee \xi_{\text {lat }}(k+1) \geq T r_{\text {.lat. }}\right) \\
& \qquad s(k)=1 \\
& \text { else } \quad s(k)=0 .
\end{aligned}
$$


(1) Input: Eucli_Dist, Max_Number_of_Repetitions, Max_Transmission_Dist,

(2) Min_Repetions_Dist, Repetions_Interval, Adjust_PDR;

(3) Output: Number_of_Repetitions;

(4) If Eucli_Dist < Max_Transmission_Dist and Eucli_Dist > Min_Repetions_Dist then

(5) If Number_of_Repetitions < Max_Number_of_Repetitions then

(6) If $P D R \leq$ Adjust_PDR

(7) Increase_Num_of_Repetions(Number_of_Repetitions, Repetions_Interval);

(8) Else

(9) Number_of_Repetitions $=$ Max_Number_of_Repetitions;

(10) End

(11) Else

(12) Decrease_Num_of_Repetions(Number_of_Repetitions, Repetions_Interval);

(13) Else

(14) Increase_Num_of_Repetions(Number_of_Repetitions, Repetions_Interval);

(15) End

Algorithm 1: The repetition model decision algorithm based on intervehicle distance.

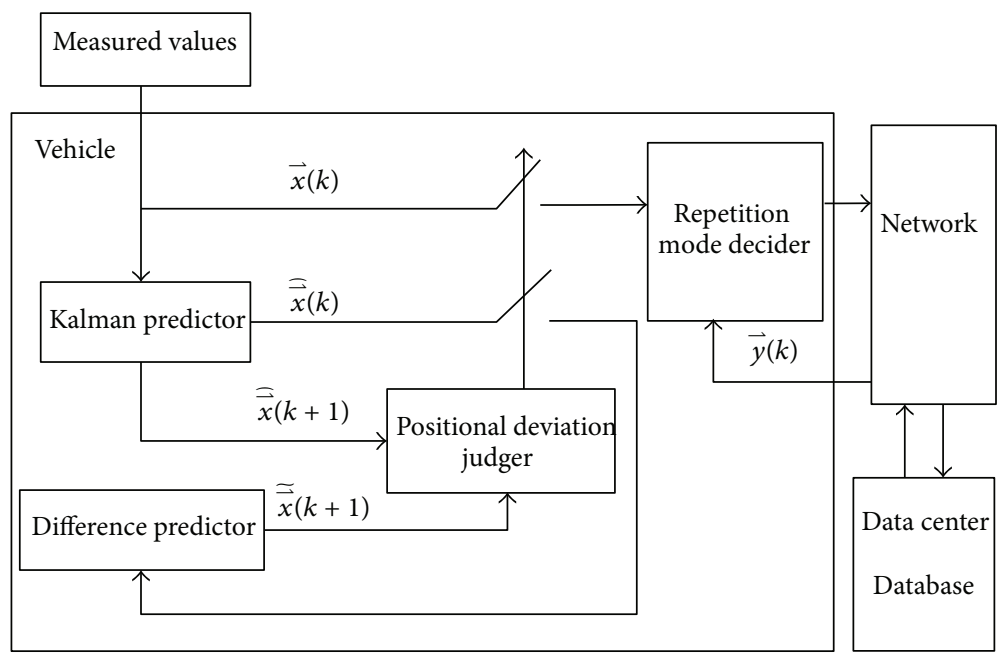

FIGURE 3: The vehicle position updating model with packet repetition.

$T r_{\text {.long. }}$ is the vertical deviation threshold. $T r_{\text {.lat. }}$ is the horizontal prediction deviation threshold. $s(k)$ is whether to send the position information in the moment $k$. "1" represents "to send" and "0" represents "not to send."

\section{Simulation Verification}

6.1. The Simulation Scheme Designing. In the road network, the frequency of vehicles position updating is related to the vehicles driving state, which is changed with vehicle speed and the direction. In order to estimate the message generation speed in a section, with reference to the meaning of [bytes $* \mathrm{~m} / \mathrm{s}$ ] that is used to measure the ability of wireless node communication in MANET (Mobile Ad Hoc Network), this paper adopts the number of position updating messages that the vehicle has generated on a certain length of the road section during a certain time window as the evaluation index (in this paper, called messages generation density, MGD). Here, we take [messages $/ 300 \mathrm{~m} * 3$ minutes] as the unit of $M G D$.
In Kalman filter, since the measured values of vehicles sensor cause background noise, so the path predicted values cause uncertainty error. In this paper, in order to simulate the output errors from the Kalman filter predictor, we use the Gauss colored noise to simulate the background noise of Kalman filter prediction. We choose colored noise instead of white noise because the experimental data shows that the Kalman filter predictor errors are colored rather than white. We use linear prediction autoregressive method to eliminate Gauss colored noise. Referring to the conclusion in literature 13 which corresponds to $X, Y, V$, and $\phi$, the mean value of Gauss colored noise is $0.2[\mathrm{~m}], 0.2[\mathrm{~m}], 0.3[\mathrm{~m} / \mathrm{s}]$, and $0.5^{\circ}$.

6.2. The Simulation Process. As shown in Figure 4, the simulation process steps are as follows.

Step 1. Select the simulation road section and determine the simulation parameters.

Step 2. Input the simulation parameters into traffic simulator to simulate and then extract all vehicles' original position 


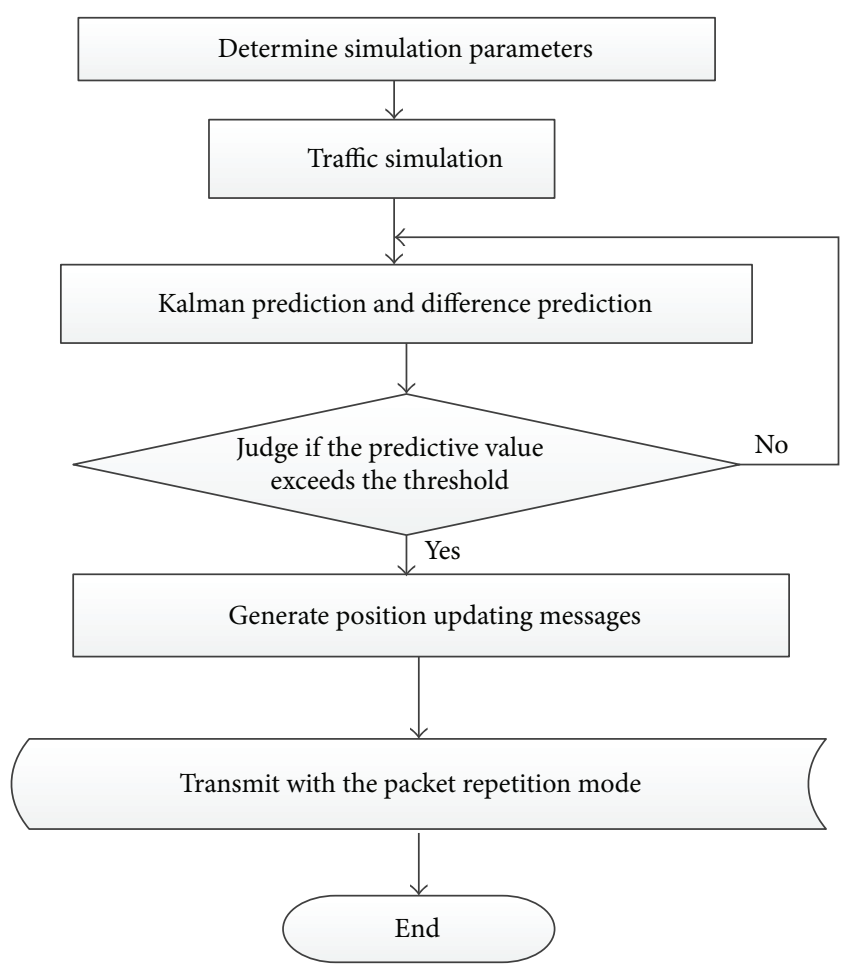

FIGURE 4: Simulation verification process.

data on the traffic simulator on this road section at a certain sampling frequency.

Step 3. Input original position data received from Step 2 into the Kalman predictor to get predicted value $\overline{\vec{x}}(k+1)$.

Step 4. Input predicted values received from Step 3 into difference predictor, namely, putting them into (6) to get difference predicted value $\underset{\vec{x}}{\widetilde{x}}(k+1)$.

Step 5. Input the values received from Steps 3 and 4 into the position deviation judgment, namely, putting them into (7) to calculate $\xi_{\text {long }}$ and $\xi_{\text {lat }}$. Then compare them with the preset deviation threshold. Finally determine whether to generate position updating messages.

Step 6. Record all the generated position updating points for subsequent analysis.

6.3. The Results and Analysis of Simulation. This paper selects an eight-lane highway basic section as the simulation object to study the messages generation of the vehicle position updating strategy and chooses Paramics as traffic simulation tools. According to experience, we take the horizontal threshold as $0.3[\mathrm{~m}]$ and the vertical threshold as $0.2[\mathrm{~m}]$. For an eight-lane highway simulation scenario, the unit of $M G D$ is [messages $/ 300 \mathrm{~m} * 3$ minutes].

Simulation parameter values are presented in Table 1 .

The average flow rate for each lane is 1800 [vph] and the average space headway is $30[\mathrm{~m}]$. Without using position updating strategy, under the condition that all vehicles'
TABLE 1: Simulation parameters on eight-lane highway basic section.

\begin{tabular}{lc}
\hline Parameter & Value \\
\hline Section length & $6[\mathrm{~km}]$ \\
Sample interval & $0.5[\mathrm{~s}]$ \\
Average traffic flow per lane & $1800[\mathrm{vph}]$ \\
Average time headway & $1.3[\mathrm{~s}]$ \\
One-way lanes & 4 \\
Minimum flow for each lane & $300[\mathrm{vph}]$ \\
Maximum flow for each lane & $2200[\mathrm{vph}]$ \\
\hline
\end{tabular}

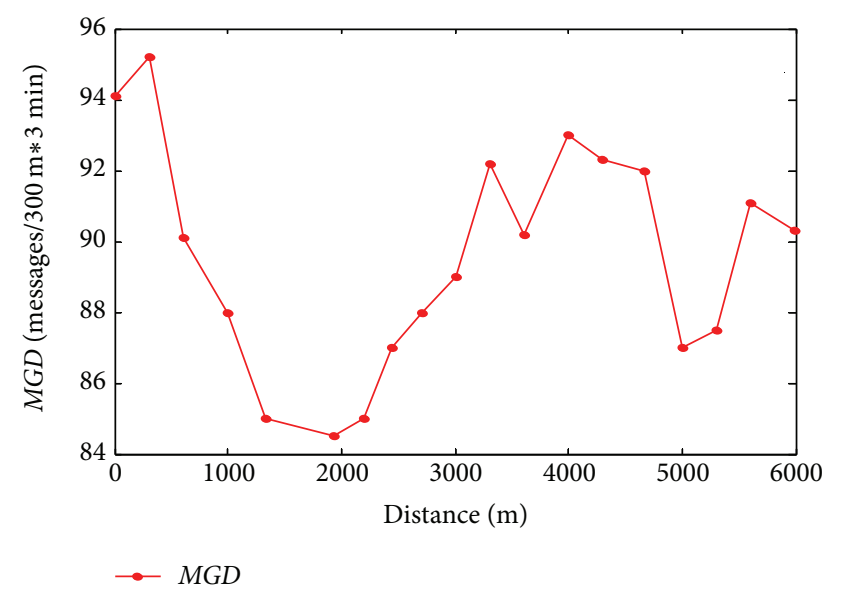

FIgURE 5: The distance-MGD—on an eight-lane highway.

periodic position updating interval is 10 [s] (i.e., six times per minute), in every $300[\mathrm{~m}]$ length of road, within three-minute window range, the number of periodic updating messages is

$$
\begin{aligned}
& \frac{300[\text { section_length }]}{30 \text { [between_cars }]} \times 4 \text { [lanes] } \\
& \quad \times \frac{180[\text { time_window }]}{10[\text { sampling_period }]}=720 \text { [messages] } .
\end{aligned}
$$

After using position updating strategy, the condition is shown in Figure 5.

As shown in Figure 5, when using position updating strategy, the number of $M G D$ is less than 100, and the highest number is 96 . When not using position updating strategy, the number of $M G D$ is 720 . So the number of vehicle positions updating request is dropped to about $1 / 7$ of the original. Thus the database refreshing rate (database I/O load) is dropped to $1 / 7$ compared with the original. So the effect is obvious, with satisfactory results.

As shown in Figure 6, the road traffic flow rate varies from 400 [vph] to 2100 [vph]. The maximum number of $M G D$ is no more than 100 . The result proves that the proposed strategy is effective.

\section{Actual Road Verification}

We utilize float car data collected on July 9, 2014, in Changchun to validate the strategy proposed in this paper. The experimental vehicle fleet consists of 15 leased taxis which 
TABLE 2: Comparison of measured messages and used strategy messages.

\begin{tabular}{lcc}
\hline $\begin{array}{l}\text { Road section } \\
\text { serial }\end{array}$ & $\begin{array}{c}\text { Measured } \\
\text { messages }\end{array}$ & Messages of used strategy \\
\hline 1 & 338 & 69 \\
2 & 421 & 71 \\
3 & 579 & 122 \\
4 & 231 & 45 \\
5 & 461 & 92 \\
6 & 296 & 56 \\
7 & 143 & 26 \\
8 & 443 & 82 \\
\hline
\end{tabular}

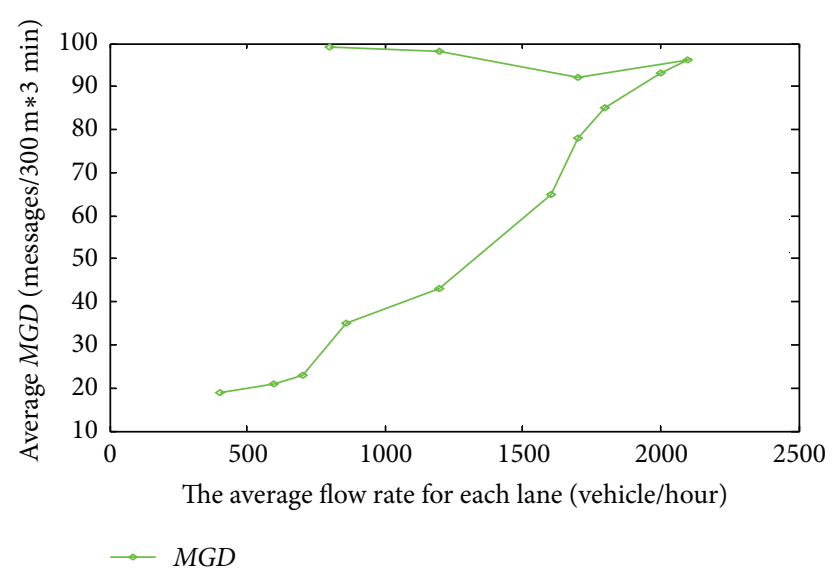

FIgURE 6: The flow rate-MGD—on an eight-lane highway.

run along the South Lake road, Yatai street, Ziyou road, and Renmin street in Changchun. The experimental fleet goes by eight sections with signal control between 7:00 and 8:00 using GPSMap76 receiver to gather vehicle position information. The vehicles of the fleet are ordered from number 1 to number 15 by the starting order. For predicting deviation values, the horizontal threshold is set to $0.3[\mathrm{~m}]$; vertical threshold is set to $0.2[\mathrm{~m}]$. At the same time, we use a stopwatch to record $t$ accurate travel time that the fleet expends through each road. Periodic position updating interval is set to 10 [s].

The numbers of position updating generation messages by using the updating strategy were compared with the numbers of measured periodic messages. The result is shown in Table 2. After applying the proposed strategy, the average number of messages is reduced to about $1 / 5$ of the original. The result is satisfactory and illustrates that this strategy is effective.

According to the preset parameters in Table 2, we compare periodic position updating messages with the using strategy position updating messages produced by the number 7 vehicle. The results are shown in Figure 7.

According to Bai and Krishnan's [21] conclusions in the urban road environment, the relationship between PDR values and the communication distance of two vehicles is positive correlation. In this paper, we set expected $P D R$ to 0.90 , the minimum transmission distances to $50[\mathrm{~m}]$, maximum repetition times to 5 , repetition interval to 0.5 [s],

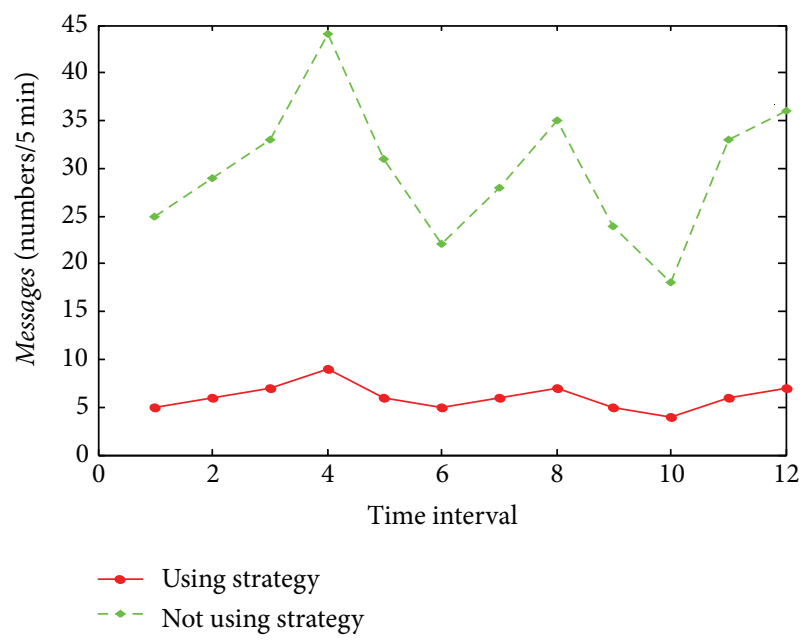

Figure 7: Messages of the 7th vehicle before and after using position updating strategy.

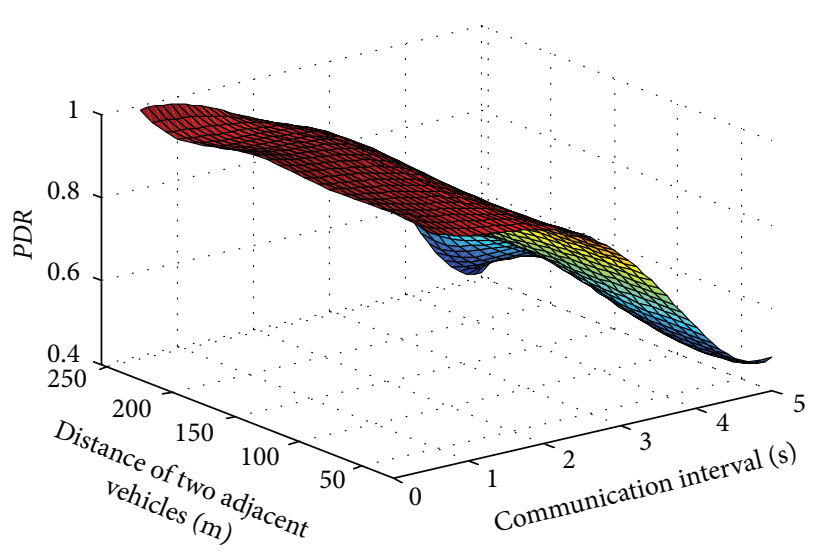

FIGURE 8: The PDR of not using repetition model decision algorithm (number 7).

and maximum transmission distances to 500 [m]. We choose American Denso Inc. V2X in-car machine which supports DSRC protocol as intervehicle communication platform. Repetition mode decision algorithm runs with above parameters. Position updating messages that are generated in numbers 7 and 8 and the communication process with the adjacent vehicles is acquired and analyzed. The $P D R$ values of using and not using repetition mode decision algorithm are shown in Figures 8 and 9.

As shown in Figure 10, when using repetition mode decision algorithm (green curve), the repetition times were adjusted dynamically so as to improve $P D R$ values effectively comparing with not using repletion model decision algorithm (red curve). Thus the reliability of message transmission is improved. The $P D R$ value after using algorithm in this experiment rises to 0.90 as above, which has achieved the expected results. So this algorithm is effective. 


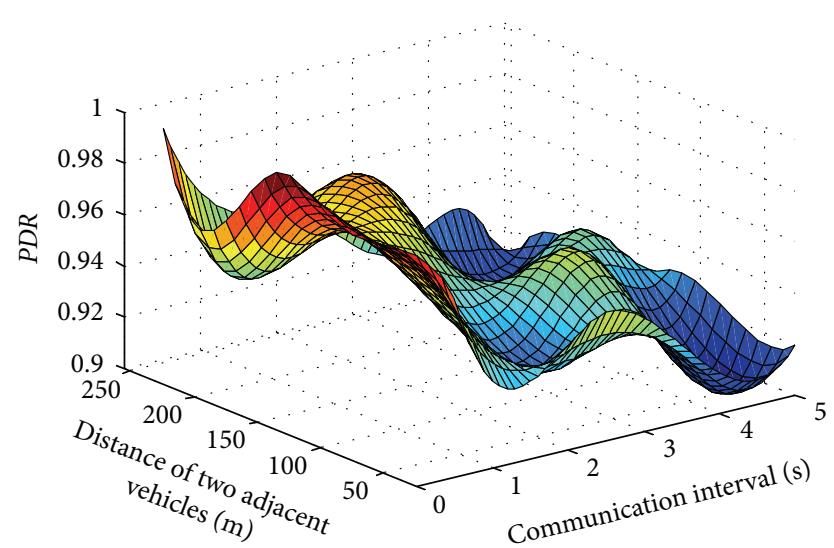

FIGURE 9: The $P D R$ of using repetition model decision algorithm (number 7).

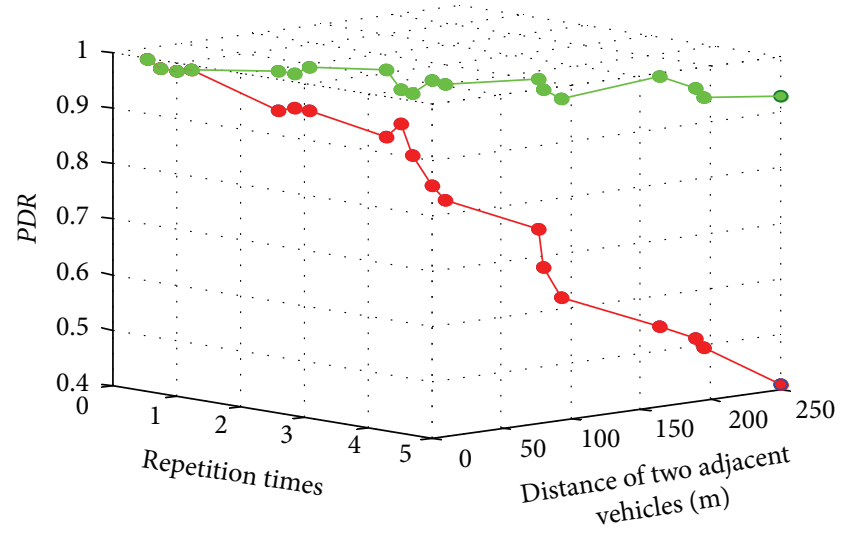

FIGURE 10: The PDR before and after using repetition model decision algorithm (number 8).

\section{Conclusions}

In VANET environment, the positions of vehicle nodes change frequently and have short cycles, which bring enormous pressure to the network communication and the data storage and updating in data center. This paper considers that the vehicle movement patterns (position, speed, and direction) can be stored in the form of dynamic vector which has implemented position updating in central MOD database. On this basis, we designed a position updating model and strategy based on Kalman filter. In order to reduce the packet loss and improve the $P D R$ over DSRC communication protocol, we designed a repetition mode decision algorithm based on the distance of two adjacent vehicles for communication. The simulation and actual road experiment show that this position updating strategy with packet repetition can significantly reduce the vehicle $M G D$ and increase PDR so as to greatly save network bandwidth, reduce the pressure of database management, and enhance the reliability of network transmission.

Here, we assumed that the wireless network transmission delay is extremely small. In fact, the presence of transmission delay always makes position updating data outdated, making the position data stored in the database unavailable. The next research will be launched on how to ensure the positional deviation error under the control in the condition of high time delay.

In this paper, the horizontal threshold and the vertical threshold were fixed at $0.3[\mathrm{~m}]$ and $0.2[\mathrm{~m}]$ according to experience. The same situation occurs in periodic position updating interval, which was set to 10 [s] in this paper. Since a vehicle position updating algorithm has to be operated well in all possible situations, future work can be conducted that addresses the issue of stability and robustness under different offset thresholds. Furthermore, consolidation efforts are required in order to provide algorithm performance comparisons of the various periodic positions' updating interval.

\section{Conflict of Interests}

The authors declare that there is no conflict of interests regarding the publication of this paper.

\section{Acknowledgment}

This work is supported by the Chinese National High Technology Research and Development Program (Grant no. 2014BAG03B03).

\section{References}

[1] H. Hartenstein and K. P. Laterteaux, Vehicular Applications and Inter-Networking Technologies, John Wiley \& Sons, Atrium, London, UK, 2010.

[2] A. Behrend, G. Schüller, and M. Wieneke, "Efficient tracking of moving objects using a relational database," Information Systems, vol. 38, no. 8, pp. 1269-1284, 2013.

[3] C. P. Wang, "Research on query mechanism of moving object database," Applied Mechanics and Materials, vol. 530-531, pp. 823-826, 2014.

[4] İ. Ayabakan and P. Kilimci, "Moving object databases-indexing algorithms," International Journal of Computer Theory and Engineering, vol. 6, no. 6, pp. 455-459, 2014.

[5] N. Meghanathan, "A location prediction based routing protocol and its extensions for multicast and multi-path routing in mobile ad hoc networks," Ad Hoc Networks, vol. 9, no. 7, pp. 1104-1126, 2011.

[6] H. Hajari and F. Hakimpour, "A spatial data model for moving object database," International Journal of Database Management Systems, vol. 6, no. 1, pp. 1-20, 2014.

[7] A. K. Gupta, G. Wable, and T. Batra, "Collision detection system for vehicles in hilly and dense fog affected area to generate collision alerts," in Proceedings of the International Conference on Issues and Challenges in Intelligent Computing Techniques (ICICT '14), pp. 38-40, IEEE, Ghaziabad, India, February 2014.

[8] Q. Wu, J. Huang, J. Luo, and J. Yang, "An all-time-domain moving object data model, location updating strategy, and position estimation," International Journal of Distributed Sensor Networks, vol. 2015, Article ID 463749, 11 pages, 2015.

[9] L. Xu, L. Y. Wang, G. Yin, and H. Zhang, "Coordinated control and communication for enhanced safety of highway vehicle platoons," in Proceedings of the 2nd IEEE International 
Conference on Connected Vehicles and Expo (ICCVE '13), pp. 4347, IEEE, Las Vegas, Nev, USA, December 2013.

[10] T. Sekine, "Utilization of probe powered two-wheeler vehicles to realize a safe mobile society," IATSS Research, vol. 38, no. 1, pp. 58-70, 2014.

[11] A. Amoroso, G. Marfia, and M. Roccetti, "Going realistic and optimal: a distributed multi-hop broadcast algorithm for vehicular safety," Computer Networks, vol. 55, no. 10, pp. 25042519, 2011.

[12] G. Marfia, M. Roccetti, A. Amoroso, and G. Pau, "Safe driving in LA: report from the greatest intervehicular accident detection test ever," IEEE Transactions on Vehicular Technology, vol. 62, no. 2, pp. 522-535, 2013.

[13] A. A. Oloufa and A. E. Radwan, "Server-based collision avoidance using DGPS," in Proceedings of 8th World Congress on Intelligent Transport System, Sydney, Australia, SeptemberOctober 2010.

[14] J. Huang and H.-S. Tan, "Vehicle future trajectory prediction with a DGPS/INS-based positioning system," in Proceedings of the American Control Conference, IEEE, Minneapolis, Minn, USA, June 2006.

[15] D.-J. Jwo, C.-F. Yang, C.-H. Chuang, and T.-Y. Lee, "Performance enhancement for ultra-tight GPS/INS integration using a fuzzy adaptive strong tracking unscented Kalman filter," Nonlinear Dynamics, vol. 73, no. 1-2, pp. 377-395, 2013.

[16] K. Jo, K. Chu, and M. Sunwoo, "Interacting multiple model filter-based sensor fusion of GPS with in-vehicle sensors for real-time vehicle positioning," IEEE Transactions on Intelligent Transportation Systems, vol. 13, no. 1, pp. 329-343, 2012.

[17] D.-C. Peng, "The basic principle and application of Kalman filter," Journal of Software Guide, vol. 8, no. 7, pp. 32-34, 2010.

[18] K. Chen and J. Yu, "Short-term wind speed prediction using an unscented Kalman filter based state-space support vector regression approach," Applied Energy, vol. 113, pp. 690-705, 2014.

[19] S. Jafarzadeh, C. Lascu, and M. S. Fadali, "State estimation of induction motor drives using the unscented Kalman filter," IEEE Transactions on Industrial Electronics, vol. 59, no. 11, pp. 42074216, 2012.

[20] X. Yang, J. Liu, F. Zhao, and N. Vaidya, "A vehicle-to-vehicle communication protocol for cooperative collision warning," in Proceedings of the Conference on Mobile and Ubiquitous Systems: Networking and Services, Boston, Mass, USA, August 2004.

[21] F. Bai and H. Krishnan, "Reliability analysis of DSRC wireless communication for vehicle safety applications," in Proceedings of the IEEE Intelligent Transportation Systems Conference (ITSC '06), pp. 355-362, IEEE, Toronto, Canada, September 2006. 


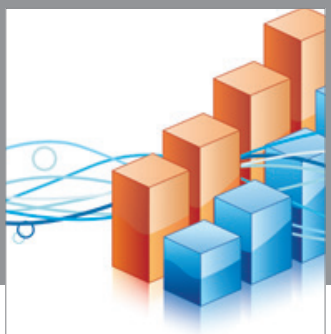

Advances in

Operations Research

vatem alat4

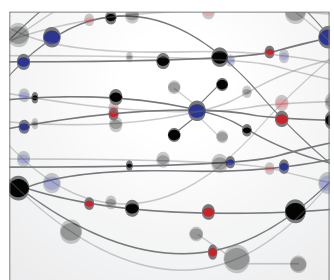

\section{The Scientific} World Journal
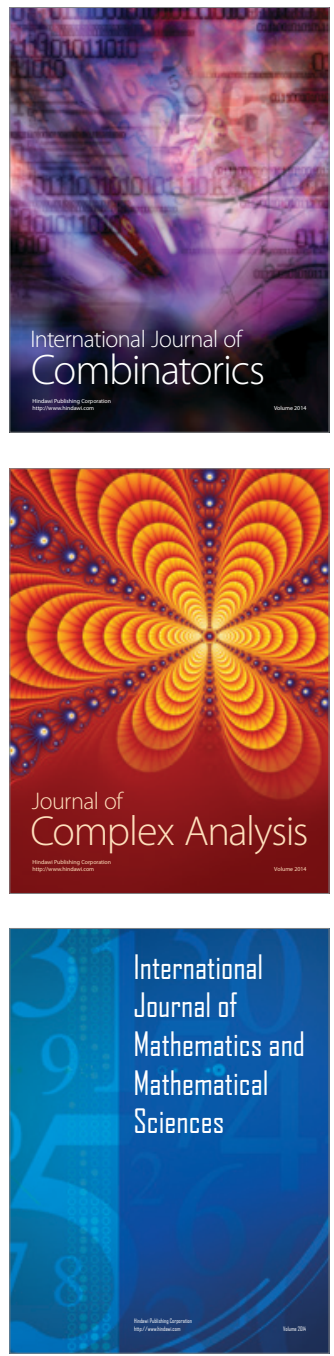
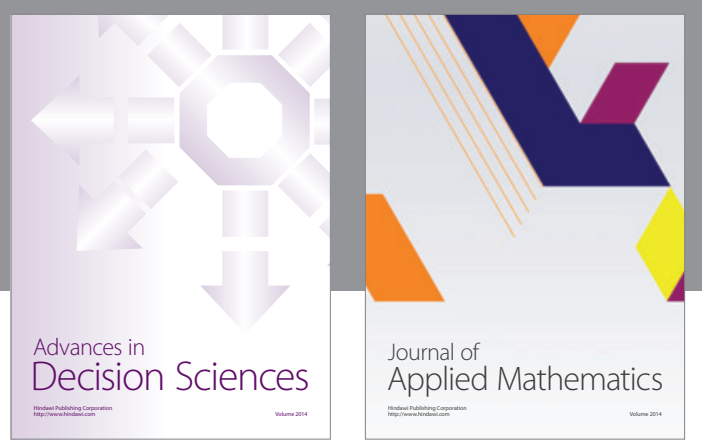

Algebra

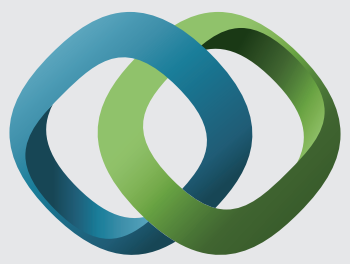

\section{Hindawi}

Submit your manuscripts at

http://www.hindawi.com
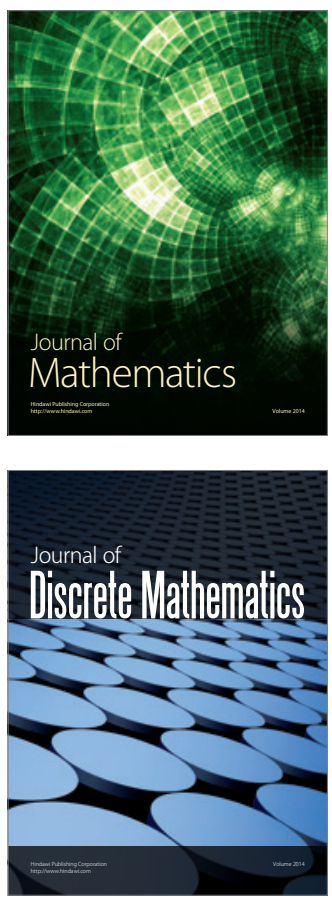

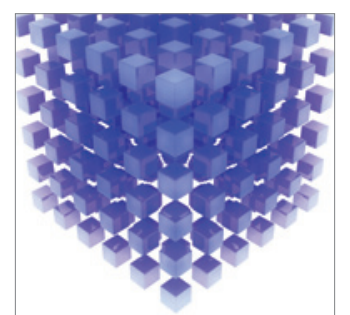

Mathematical Problems in Engineering
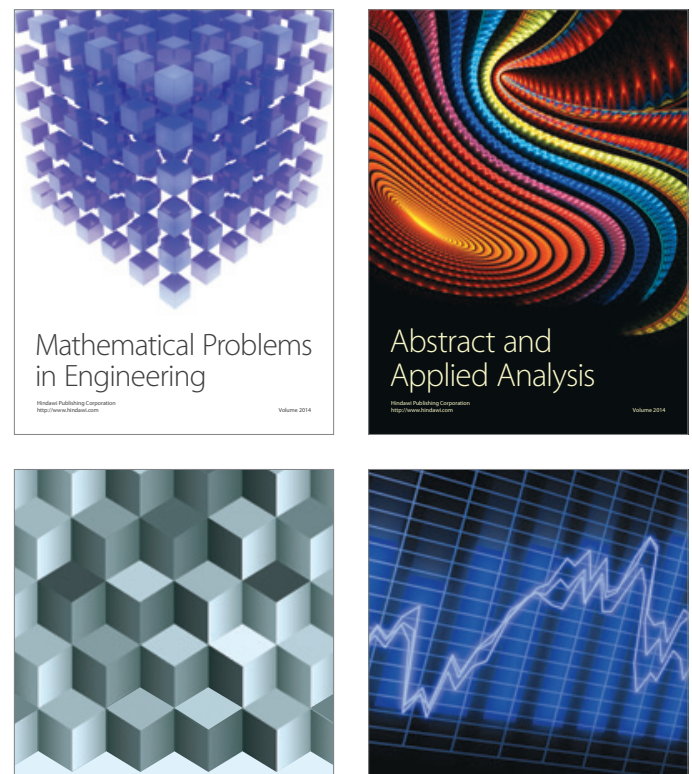

Journal of

Function Spaces

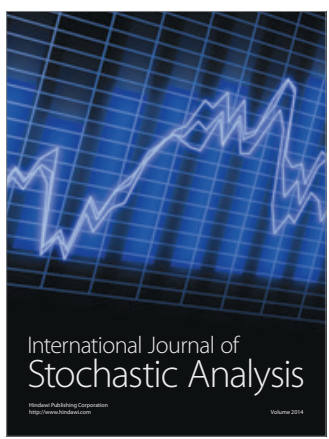

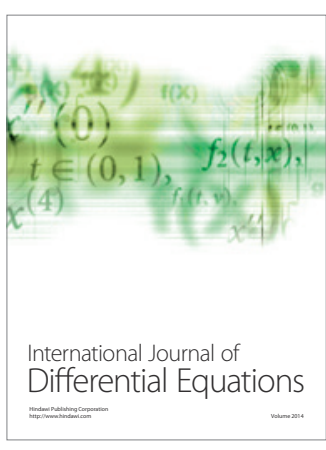
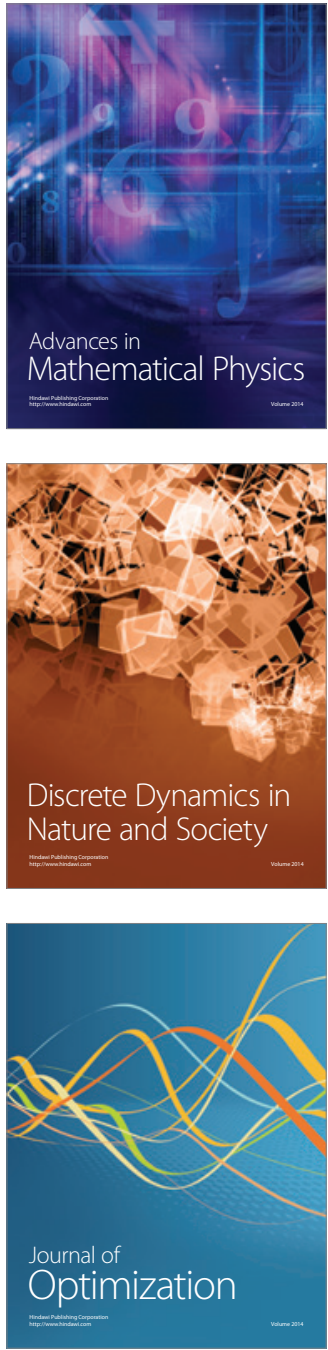